\title{
Extensively and partially hydrolysed infant formulas for allergy prophylaxis
}

\author{
G Oldæus, K Anjou, B Björkstén, J R Moran, N-I M Kjellman
}

Department of

Paediatrics,

Länssjukhuset Ryhov, Jönköping, Sweden

G Oldæus

Department of Paediatrics, University Hospital, Linköping, Sweden

G Oldæus

B Björkstén

N-I M Kjellman

Department of

Paediatrics,

Länssjukhuset,

Kalmar, Sweden

K Anjou

Mead Johnson

Nutritional Group, Evansville, Indiana, USA

J R Moran

Correspondence to: Dr Göran Oldæus,

Department of Paediatrics, Länssjukhuset Ryhov, S-551 85 Jönköping, Sweden.

Accepted 17 March 1997

\begin{abstract}
The allergy preventive effect of extensively (N) and partially (PH) hydrolysed cows' milk formulas compared with a regular formula (RM) was assessed in 155 infants with a family history of allergy. No cows' milk was given during the first nine months of life and no egg and fish up to 12 months of age. Breast feeding mothers avoided the same foods. At weaning the infants were randomised to one of the formula groups. The cumulative incidence of atopic symptoms at 18 months was 51,64 , and $84 \%$ in the $N, P H$, and $R M$ groups, respectively. From 6 to 18 months there were significantly less cumulative atopic symptoms in the $\mathbf{N}$ group compared with the RM group, and significantly less than the PH group up to $6(\mathrm{~N}=25 \% ; \mathrm{PH}=46 \%)$ and 9 months $(\mathrm{N}=34 \%, \mathrm{PH}=58 \%)$. At 9 months significantly fewer infants in the $\mathbf{N}$ group (10\%) than in the PH group (33\%) had a positive skin prick test to eggs. The findings support an allergy preventive effect of an extensively hydrolysed formula, but not of a partially hydrolysed formula, during the first 18 months of life of high risk infants.

(Arch Dis Child 1997;77:4-10)
\end{abstract}

Keywords: protein hydrolysates; cows' milk allergy; allergy prevention; allergic sensitisation

Atopic disease is increasing in industrialised and developing countries. ${ }^{1}$ In Sweden one third of all infants and children up to 11 years of age have atopic disease. ${ }^{2}$ The risk of allergy is determined by interactions between genetic and environmental factors. ${ }^{3-5}$ Allergy prevention should be targeted primarily at infants with a genetically determined susceptibility. ${ }^{67}$ Preventive measures have included promoting breast feeding of infants and avoiding inhalant and potent food allergens and irritants. ${ }^{9-15}$ Some workers have included preventive measures during pregnancy, ${ }^{11}{ }^{13}$ but dietary restrictions during pregnancy have not prevented allergy in the offspring and might even be nutritionally harmful. ${ }^{13} 14$ Small amounts of dietary allergens may reach the child through the mother's breast milk, thus triggering the sensitisation, ${ }^{16-19}$ and the elimination of highly allergenic foods during the first three months of breast feeding reduced the incidence of atopic dermatitis in infants. ${ }^{9}$

Most preventive studies have included the use of a formula based on hydrolysed cows' milk for early infant feeding. Extensively hydrolysed formulas differ from regular formulas in smell and taste and are expensive. To overcome these problems less hydrolysed formulas have been introduced and there are claims that they have allergy preventive properties, ${ }^{20}$ but studies apparently showing such an effect have had flaws in their design. ${ }^{21}$

To evaluate properly any prophylactic effect of a hypoallergenic formula, randomised, preferably double blind placebo controlled, studies are needed. A position paper from the European Society of Pediatric Allergy and Clinical Immunology on hydrolysed cows' milk formulas recommended studies comparing partial hydrolysates with extensive hydrolysates. ${ }^{21}$ Our study aimed to compare the incidence and severity of atopic disease and allergic sensitisation during the first 18 months of life in infants at risk who were fed either an extensively hydrolysed cows' milk formula, a partially hydrolysed formula, or a regular cows' milk formula from the start of weaning until 9 months of age.

\section{Methods}

STUDY DESIGN

Families with a history of previous or present allergic disease who attended the well mother clinics in three towns in the southeast of Sweden (Jönköping, Kalmar, and Linköping) were invited to participate in the study. If they agreed, a study nurse conducted a structured telephone interview to confirm a family history of allergic disease. Asthma, allergic rhinitis, and atopic dermatitis diagnosed by a doctor were accepted as significant allergic disease. Food allergy was accepted if there had been immediate reactions on intake and either IgE antibodies could be shown or there was a positive oral challenge. Urticaria alone was not considered as an allergic disease unless there were also $\operatorname{IgE}$ antibodies to the suspected allergen. 
During their last trimester pregnant women were informed by a dietitian about the diet prescribed for them and their infants. One week before the expected date of delivery the mothers eliminated all sources of cows' milk, eggs, and fish from their diet. This diet was maintained throughout breast feeding. All mothers were given $1 \mathrm{~g} /$ day calcium supplements during the diet period.

Newborn infants in families with at least two family members with significant atopic disease were directly included in the study, whereas infants with single heredity were only included if their cord blood IgE concentration was at least $0.5 \mathrm{kU} / 1$. The exclusion criteria included an obvious risk for non-compliance with diet or follow up, birth defects, severe chronic disease, gestational age less than 35 full weeks, and need for mechanical ventilation. Our intention was to obtain a study population with an expected risk of developing allergic disease during the first 18 months of life of at least $40 \%$. Assuming that the programme would give a $25 \%$ reduction in allergic disease, we estimated that we required 55 subjects in each group to detect a difference with $80 \%$ probability.

All families were recommended to take general allergy preventive measures, such as not smoking and avoiding furry animals at home, in concordance with the Swedish National Program for Allergy Risk Families. Breast feeding was encouraged.

At birth, a cord blood sample was taken and a nurse provided further information about the study. A tin of hypoallergenic formula $(\mathrm{Nu}-$ tramigen, Mead Johnson, Evansville, Indiana, USA), was provided to all participants to use if they started weaning before randomisation. Parents completed a simple diary registering symptoms and events and the dates they introduced formula and other foods during the first 12 months. The infants' dietary restrictions were as follows: foods containing milk were not to be introduced during the formula feeding period of nine months; eggs, fish, and citrus fruits were not to be introduced during the first year of life; and other solid foods were only allowed from 4 months of age.

When weaning started the infants were randomised to one of three formula groups: a partially hydrolysed formula, PH (Mead Johnson), an extensively hydrolysed casein formula, $\mathrm{N}$ (Nutramigen, Mead Johnson), or a routine cows' milk formula, RM (Enfamil, Mead Johnson). The PH formula had a protein, fat, and carbohydrate content similar to that of RM. Protein was provided by a 60:40 whey to casein blend which had undergone mild enzymatic hydrolysis to reduce the antigen content. Carbohydrate was provided by lactose, as in routine milk formulas. The fat was a blend of soy, corn, and coconut oils. Randomisation was stratified according to the age at starting weaning/formula introduction - that is, $0-<2$, $2-<4,4-<6$, and $6-<9$ months of age. Within these groups infants were randomly assigned to one of the formulas. The purpose of the age grouping was to assure a similar weaning age in each formula group. Infants received the study formula free of charge until 9 months of age. The formula tins were coded and there was no difference in design. No attempt was made to mask the cows' milk formula. Nurses and investigators, however, were blinded throughout the study. Twenty one of the mothers chose to breast feed their infants for more than 9 months so they were never randomised. The results from this group are presented as group HM.

FOLLOW UP

The infants were seen by a nurse at $3,6,9,12$, and 18 months of age to document growth, formula acceptance and tolerance, and clinical symptoms. Interviews used a structured form. We carried out a detailed evaluation of infants with atopic dermatitis using a scoring system by which the body surface was divided into 20 areas. All areas were classified according to redness, excoriations, lichenification, and vesiculation by a four degree scoring system from zero to three. ${ }^{22}$ The sums of these scores were then used in the statistical calculations.

Based on the clinical symptoms as documented at interviews and examinations at 3, 6 , 9,12 , and 18 months, and the double blind placebo controlled challenges, each infant was classified according to the presence or absence of atopy or a specific atopic symptom.

If allergic disease occurred other than a reaction to food, infants were treated according to usual practice by their own doctor. We encouraged those families who decided not to complete the study protocol to continue the follow up programme.

\section{CHALLENGE PROCEDURES}

We performed double blind placebo controlled challenges in infants suspected of having a reaction to formula milk if the reaction was not systemic. Coded samples of the formulas for these challenges were delivered by the manufacturer. The control/placebo formula was Nutramigen. The other challenge formulas were all mixed 3:1 with Nutramigen to make them similar in smell and taste. The randomised formula was replaced by Nutramigen for two weeks before the challenge as a washout procedure. The challenges were performed in random order at the doctor's surgery, starting with $1 \mathrm{ml}$ and increasing the dose to 5, 10, 50, and $100 \mathrm{ml}$ at 30 minute intervals. If no reaction was seen within one hour after the last dose, the child was taken home and feeding was started with the challenge formula and continued for one week. If no symptoms occurred during this period then the challenge was considered to be negative. After a two week washout period with Nutramigen a second challenge was performed in the same manner. If the challenge was positive we considered that the child had completed the study.

In children who were sensitised to a certain food, open challenges were performed at the doctor's surgery with the specific food after the end of the diet period. This was carried out to decide whether the child was able to eat the indicated food-for example, cows' milk after 9 months and eggs after 12 months of age. The 
milk challenges were carried out in an open setting under the supervision of a doctor and a nurse. The starting dose was $0.1-1 \mathrm{ml}$, then 5 , 10,25 , and $50 \mathrm{ml}$ were given every 30 minutes. If there was no reaction within one hour of the last dose, the child was allowed to take a regular cows' milk formula at home. If a reaction occurred during the challenge or within one week of the start of feeding, the child was considered to be allergic to cows' milk.

Open challenges with hens' eggs were carried out, starting with $1 \mathrm{~g}$ of sponge cake and ending with $10 \mathrm{~g}$ of pure egg white. The results were assessed as just described.

FINAL EVALUATION

One investigator (GO) saw all the children at 18 months of age and made a final cumulative diagnosis based on the previously collected clinical data from the study, the skin prick test results, and a final clinical examination. No laboratory data was available at this time, with the exception of cord blood IgE.

We diagnosed asthma if the child had wheezed on at least three occasions, at least one of which had been verified by a doctor. If the parents reported that wheezing had occurred, but there was no verification by a doctor, then we classified diagnosis as probable.

We defined atopic dermatitis as typical itching skin lesions ${ }^{23}$ present on at least one visit. If the lesions were not verified at the visits, but only described by parents as episodes of itching skin lesions between visits, we classified atopic dermatitis as probable.

Gastrointestinal allergy was obvious if verified by a positive oral challenge and probable if there was merely a history of a reaction in combination with a positive skin prick test to the food, but no oral challenge was carried out.

Obvious allergic rhinitis and conjunctivitis were accepted if a clinical reaction was verified by a doctor and sensitisation was proved to the suspected allergen, otherwise it was categorised as probable.

We categorised children as having obvious atopic disease if there was at least one obvious diagnosis of asthma, atopic dermatitis, allergic rhinoconjunctivitis, or gastrointestinal allergy. Children with no obvious or probable atopic manifestations were categorised as having no atopic disease.

TESTS FOR IGE ANTIBODIES

Cord blood IgE levels were determined using Phadebas IgE PRIST (Pharmacia, Uppsala, Sweden) in a modified version of the original procedure, with a detection limit at $0.1 \mathrm{kU} / 1 .^{24}$ At 9,12 , and 18 months a venous blood sample was drawn and analysed for IgE antibodies to eggs and cows' milk (CAP System RAST RIA, Pharmacia). Detectable values were considered positive. At 18 months a screening test for IgE antibodies against a panel of allergens was also used (Phadiatop Paediatric RIA, Pharmacia). The CAP RAST is a semiquantative assay with the detection limit set at 0.35 $\mathrm{kU} / \mathrm{l}$, whereas the Phadiatop Paediatric test is qualitatitve, giving either a positive or a negative result.
Skin prick tests were carried out at each visit, beginning at 3 months. Fresh cows' milk with a fat content of $0.5 \%$, natural hens' egg white, cod fish, and raw potato were used as allergen sources. At 9, 12, and 18 months mites (Dermatophagoides pteronyssinus) and animal dander (dog, cat) were added to the test panel (Phazet, Pharmacia). During the feeding period the blinded formulas were always tested in duplicate in a 'ready to drink' solution. Histamine $(10 \mathrm{mg} / \mathrm{ml})$ was used as a positive control. The largest diameter of the weal and the diameter perpendicular to this were measured and the mean of these measurements was recorded. A skin prick test with a mean diameter of $3 \mathrm{~mm}$ or more was considered to be positive. ${ }^{25}$

\section{STATISTICAL METHODS}

Differences in birth anthropometric data and changes in anthropometric measurements from birth were subject to analysis of variance. Subject characteristics, environmental conditions, and discontinuation rates stratified by study site were analysed by the CochranMantel-Haenszel test and by analysis of variance. The Kruskal-Wallis test was used to analyse the cumulative and period prevalence of symptoms when the response had more than two levels, otherwise Fisher's exact test was used. The cumulative and period prevalence of positive skin prick tests as well as the Phadiatop Paediatric results and serum IgE data (dichotomised response) were analysed using Fisher's exact test and continuous data by the KruskalWallis test using a $\chi^{2}$ approximation.

The Kruskal-Wallis exact test was used to analyse the final cumulative diagnosis at 18 months and the Kruskal-Wallis test with a $\chi^{2}$ approximation was used to analyse the number of non-allergic events from the diaries. All calculations of $p$ values were based on two tailed tests. All tests were conducted at an alpha level of 0.05 . If the overall test was significant, pairwise comparisons were made.

ETHICS

The study was approved by the human research ethics committee at the University Hospital, Linköping. All families were given verbal and written information and they gave written consent before enrolment in the study.

\section{Results}

A total of 304 families with single or double heredity for atopy was enrolled. Infants with single heredity and cord blood IgE concentrations $<0.5 \mathrm{kU} / 1$, a total of 101 , were not included and were not followed up. Thus 203 infants were included in the study. Twenty seven infants were withdrawn before randomisation, almost all due to difficulties in complying with the maternal diet. Another 21 infants were breast fed for more than nine months and were thus not randomised, leaving 155 infants to be randomised: 55 to Nutramigen $(\mathrm{N}), 51$ to the partially hydrolysed formula $(\mathrm{PH})$, and 49 to Enfamil (RM). Of the 155 infants who were randomised to a study formula, 14 discontinued the study: five $(9 \%)$ in the $\mathrm{N}$ group, six 


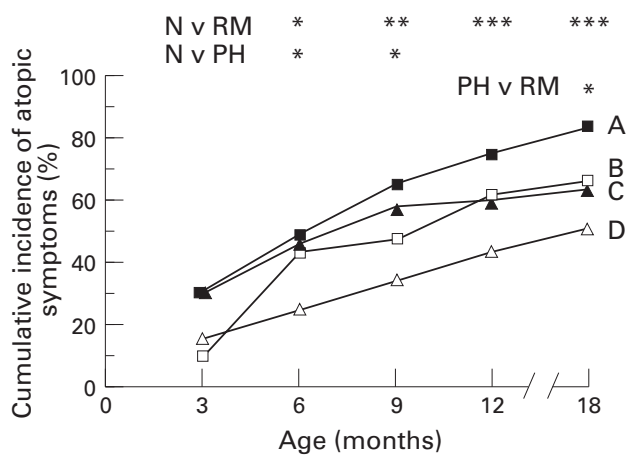

Figure 1 Cumulative prevalence of atopic symptoms in infants receiving $(A)$ routine milk formula $(R M),(B)$ those who were fully breast fed for more than nine months, (C) those who received partial hydrolysate $(\mathrm{PH})$, or (D) those who received Nutramigen $(N)$. The levels of significance are indicated in the upper part of the figure.

$(12 \%)$ in the $\mathrm{PH}$ group, and three $(6 \%)$ in the RM group (NS). The reason was feeding problems in 11 infants (three, six, and two in the N, $\mathrm{PH}$, and RM groups, respectively), moving from the region (two infants), and maternal disease (one infant). Thus 141 of 155 children (91\%) attended the final follow up at 18 months - that is, 50, 45, and 46 in the N, PH, and RM groups, respectively. All 21 children who were breast fed for nine months or more were followed up (HM group).

Of the 176 children who were either randomised to formula or breast fed for nine months, $97(55 \%)$ were boys and 79 (45\%) were girls. The birth weights were similar in the four groups and between the boys and girls (data not shown). Between 73 and 84\% (NS) of the families in each group had two or more family members with a history of atopic disease. Smoking at home occurred in 16-20\% (NS) in the four groups. Furry animals were present in the homes of $22,6,16$, and $24 \%$ (NS) of the subjects in the N, PH, RM, and HM groups, respectively. At the end of the first year the corresponding percentages were 27,8 , 16 , and $24 \%$ (NS). The mean age of formula introduction was 3.6, 3.8, and 3.3 months (NS), and the mean age when weaning was completed was 5.1, 5.6, and 5.1 months (NS) in the $\mathrm{N}, \mathrm{PH}$, and $\mathrm{RM}$ groups, respectively.

The cumulative incidence of atopic symptoms was significantly lower in the $\mathrm{N}$ than the $\mathrm{RM}$ group at $6,9,12$, and 18 months $(\mathrm{p}=$ $0.013-<0.001)$ and lower than in the $\mathrm{PH}$ group at $6(p=0.025)$ and 9 months $(p=0.018)$ (fig 1 ). The number of children with atopic symptoms was lower in the $\mathrm{PH}$ group than in the $\mathrm{RM}$ group at 18 months $(\mathrm{p}=0.039)$. During the first 9 months atopic symptoms were present in $34,58,65$, and $48 \%$ in the $\mathrm{N}, \mathrm{PH}$, RM, and HM groups, respectively. The corresponding figures were $51,64,84$, and $67 \%$ for the 18 month period.

Wheezing during the first 18 months of life was recorded in 13,16,33, and $5 \%$ of infants in the N, PH, RM, and HM groups (fig 2). The cumulative incidence was significantly higher in the RM group than in the $\mathrm{N}$ and HM groups ( $\mathrm{p}=0.031$ and 0.014 , respectively), whereas the difference from the PH group did not reach

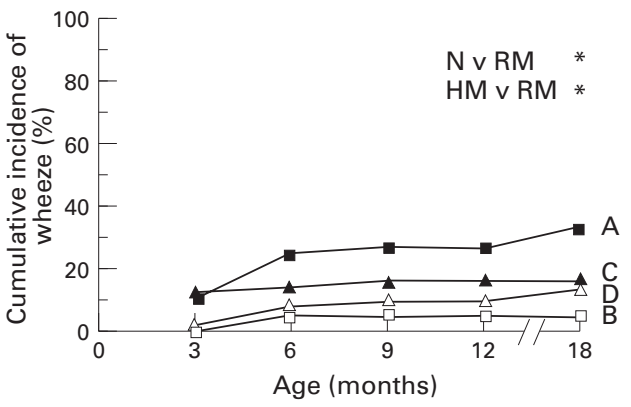

Figure 2 Cumulative prevalence of wheezing episodes. Formula groups and levels of significance as in fig 1 .

significance $(p=0.063)$. There was a similar non-significant trend at 6,9 , and 12 months of age.

In the $\mathrm{N}$ group, $17 \%$ of the children had developed atopic dermatitis by 9 months of age compared with $44 \%$ in the $\mathrm{PH}(\mathrm{p}=0.004)$ and $41 \%$ in the $\mathrm{RM}$ groups ( $\mathrm{p}=0.006$ ) (fig 3 ). Although not statistically significant, a similar pattern was seen at 6,12 , and 18 months. The eczema scores, representing the severity of eczema, were similar in the four groups at all ages.

The period prevalence of atopic symptoms at $3,6,9,12$, and 18 months was similar in all four groups, both with regard to general classification and individual atopic symptoms (fig 4). The number of atopic infants in the $\mathrm{N}$ group at 9 months was approximately half that of the other two formula groups, however.

The proportions of children with a final cumulative diagnosis of atopy at 18 months were similar in the four groups. Obvious atopic disease was recorded in $29,44,33$, and $29 \%$ in the $\mathrm{N}, \mathrm{PH}, \mathrm{RM}$, and HM groups. Including probable disease, the corresponding proportions were $38,53,59$, and $43 \%$ (NS). Obvious asthma appeared in four ( $8 \%)$, two $(4 \%)$, six $(13 \%)$, and none of the children, whereas including probable disease the proportions were $14,17,25$, and $5 \%$ in the N, PH, RM, and HM groups, respectively (NS). Atopic dermatitis was obvious in $13(25 \%), 14(29 \%), 11$ $(22 \%)$, and five $(24 \%)$ children (NS), and gastrointestinal allergy in five $(10 \%), 10(21 \%)$, four $(8 \%)$, and two $(10 \%)$ children (NS) in the $\mathrm{N}, \mathrm{PH}, \mathrm{RM}$, and HM groups, respectively. One child in the $\mathrm{N}$ group had an allergic rhinoconjunctivitis classified as probable.

An increased incidence of asthma at 18 months was seen in children exposed to smoking at home compared with non-exposed children $(p=0.006)$. Pet animals at home were, however, not associated with an increased incidence of asthma at this age. Growth was normal and similar in all the groups throughout the study period (data not shown).

A positive skin prick test to eggs was significantly more common at 9 months in the $\mathrm{PH}$ than in the $\mathrm{N}$ group ( $\mathrm{p}=0.006)$-that is, five $(10 \%), 15(33 \%), 10(22 \%)$, and three $(15 \%)$ in the $\mathrm{N}, \mathrm{PH}, \mathrm{RM}$, and HM groups, respectively. No other significant difference was seen in the period prevalence of positive skin prick tests. Nine children had positive skin prick tests to the regular milk formula during the 9 month 
Table 1 No (\%) of infants sensitised to eggs and milk as determined by CAP-RAST at the end of the formula feeding period (9 months), at the end of the study (18 months), and Phadiatop Paediatric positive subjects at 18 months. No significant differences were seen between groups

\begin{tabular}{lllll}
\hline Formula: & $N$ & $P H$ & $R M$ & $H M$ \\
\hline 9 months & $5(12)$ & $7(18)$ & $5(12)$ & $3(19)$ \\
Eggs & $1(2)$ & $1(2)$ & $5(12)$ & $1(6)$ \\
Milk & $5(11)$ & $6(15)$ & $4(10)$ & $5(24)$ \\
18 months & $5(11)$ & $2(5)$ & $7(18)$ & $1(5)$ \\
Eggs & $18(40)$ & $16(39)$ & $13(35)$ & $6(29)$ \\
Milk & Phadiatop & & & \\
\hline
\end{tabular}

$\mathrm{N}$, Nutramigen; $\mathrm{PH}$, partial hydrolysate; RM, routine milk; and $\mathrm{HM}$, breast fed $\geqslant 9$ months.

feeding period: five in the $\mathrm{N}$ group, two in the $\mathrm{PH}$ group, and two in the HM group. No child reacted to Nutramigen and two children reacted to the partial hydrolysate. A positive skin prick reaction was recorded at least once in 44 children to eggs, in 10 to milk, in 11 to potato, in three to fish, and in none to animal dander or mite. There was no significant difference between the groups in the cumulative prevalence of positive skin prick tests at any time (data not shown).

A total of 140 serum samples was available for IgE antibody analysis at 9 months, 130 at 12 months, and 147 at 18 months of age. The prevalence of IgE antibodies to eggs and milk was similar in the four groups at all ages and there was no difference in the outcome of the screening test for IgE antibodies at 18 months (table 1).

Double blind placebo controlled oral formula challenges were positive in two of three challenged infants from the RM group and one of two from the $\mathrm{PH}$ group. There was no symptom suspected to be related to formula leading to challenges in the $\mathrm{N}$ group. No reaction was seen during the challenge procedure in the hospital. In the RM group one infant had

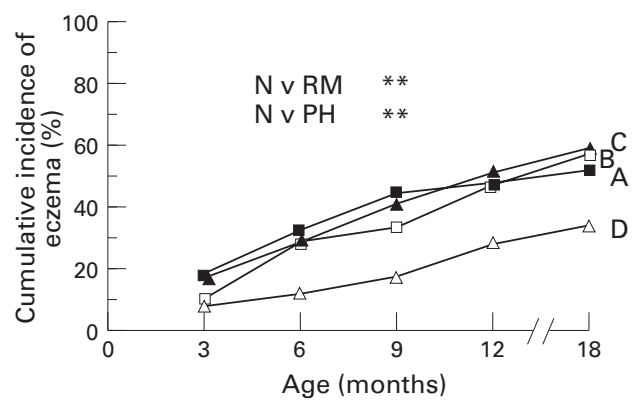

Figure 3 Cumulative prevalence of atopic dermatitis. Formula groups and levels of significance are as in fig 1 .

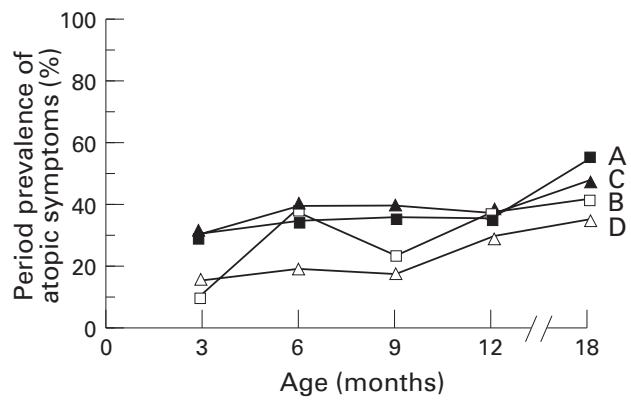

Figure 4 Period prevalence of atopic symptoms. Formula groups are as in fig 1. No significant difference was seen between the groups. a reappearance of colic within 24 hours of the start of feeding the challenge formula. The other infant had a deterioration in eczema within the first 48 hours of the start of the challenge. The infant from the $\mathrm{PH}$ group had an itching eczematous skin reaction appearing $8-10$ hours after the start of the challenge. No infant reacted to the placebo.

Open food challenges to milk were conducted in 15 infants between 9 and 12 months of age due to suspected sensitisation at the end of the diet period. A positive outcome was recorded in $3 / 6,2 / 4,2 / 4$, and $1 / 1$ in the $\mathrm{N}, \mathrm{PH}$, RM, and HM groups, respectively.

Open food challenges to eggs were conducted between 12 and 20 months of age in 24 subjects who were skin prick positive to eggs at the 12 month visit (that is, the end of the egg elimination period). The delay in some challenges was due to practical reasons or to skin prick reactions $>10 \mathrm{~mm}$. A positive challenge was recorded in $3 / 5,6 / 10,1 / 5$, and $2 / 4$ infants in the $\mathrm{N}, \mathrm{PH}, \mathrm{RM}$, and HM groups, respectively.

\section{Discussion}

In this prospective randomised blind study comparing the allergy prophylactic effect of an extensively and a partially hydrolysed cows' milk formula in high risk infants, the extensively hydrolysed formula seemed to be superior to the partly hydrolysed formula. The cumulative incidence of atopic symptoms at the end of the follow up period was lower in the $\mathrm{N}$ group than in the other formula groups. Furthermore, significantly fewer wheezing episodes were recorded in the $\mathrm{N}$ and HM groups compared with the RM group; the cumulative incidence of atopic dermatitis was significantly lower in the $\mathrm{N}$ group than in both the $\mathrm{PH}$ and $\mathrm{RM}$ groups at 9 months with a similar, but non-significant, trend up to 18 months. The $\mathrm{PH}$ group had more sensitisation to eggs at 9 months than any other group. With the exception of a moderate reduction in the cumulative incidence of atopic symptoms in the $\mathrm{PH}$ group compared with the RM group after 18 months, partial hydrolysate and regular formula were similar in the outcome of allergy.

The cumulative number of infants with obvious atopic disease at the follow up at 18 months was similar in the four groups. When adding the diagnosis of probable disease ${ }^{26}$ there was a tendency in favour of the group fed with Nutramigen. Owing to dropouts and the fact that 21 mothers chose to breast feed their infants for nine months or more we were not able to reach the anticipated number of subjects for follow up: 55 per group for an $80 \%$ power to detect a $25 \%$ reduction of allergic disease in the intervention group from the expected $40 \%$. Furthermore, the cumulative incidence of atopic disease observed in the study was actually higher and the reduction smaller than expected: $60 \%$ in the routine milk group compared with $40 \%$ in the Nutramigen group. To detect such a difference the size of each group should have been 107 for an $80 \%$ 
power. Therefore a larger study population might have revealed differences.

It is obvious from the data in this study that most of the differences in morbidity emerge during the first 3-6 months, indicating that this is the most important age for the avoidance of highly allergenic foods, as has been observed by others. ${ }^{27} 28$

The analysis of confounding factors did not reveal any difference between the groups and study sites. It is, however, difficult to perform a truly double blind feeding study. Although the tins were coded and blinded, the smell and taste of the formulas were not masked. As the physical characteristics of a non-hydrolysed and a hydrolysed formula are not identical, it might have been possible for some parents to identify the regular formula, but probably not to distinguish between the two hydrolysed formulas.

In all allergy intervention studies there are difficulties with the diagnostic definitions as they are to some extent subjective. To overcome this we tried to give as strict definitions as possible and included oral challenges for the diagnosis of gastrointestinal allergy. Formula related reactions were evaluated double blind and placebo controlled. Furthermore, the evaluation of the final diagnosis was carried out by only one of the investigators.

Partly hydrolysed formulas have various amounts of remaining antigenic determinants. The amounts are often enough to elicit immunological reactions in children allergic to cows' milk, ${ }^{29}$ and even to induce anaphylactic reactions on intake. ${ }^{30}$ In this study one child reacted to the partly hydrolysed formula during the feeding period and was later positive in the double blind placebo controlled challenge.

The protocol of this study was designed to look at the effect of eliminating one major allergen - cows' milk. We could not find differences in milk sensitisation that could explain the outcome, however. This might be due to the method used for cows' milk IgE detection. We have indications that a method with a higher sensitivity would have shown such differences (Oldæus, unpublished data). Another possibility is that early exposure to a highly allergenic food such as cows' milk primes the immune system and induces allergic disease, although the cows' milk allergy in itself is not apparent.

The only difference in sensitisation in this study was seen for eggs, which was most common in the $\mathrm{PH}$ group. This finding is interesting as early egg sensitisation has been suggested as a predictive marker for subsequent allergic disease. ${ }^{24}$ There could have been group specific differences in compliance with the diet, but such differences were not actually seen. We have no indications that, for instance, egg was added differently in the groups. In fact, 76, 81, 67 , and $82 \%$ (NS) of the mothers in the HM, $\mathrm{N}$, RM, and $\mathrm{PH}$ groups, respectively, stated that their infants kept to the diet during the first 12 months of life. Another possibility is that new epitopes, maybe of importance for egg sensitisation, could have developed during the partial hydrolysis process.
Recent allergy preventive studies concentrating on food allergen avoidance have not shown any effect on asthma. ${ }^{10-12}$ On the other hand, exclusive breast feeding for three months or more has been shown to reduce the risk of admission to hospital related to asthma. ${ }^{31}$ Furthermore, in a 17 year follow up study of infants at normal risk, exclusive breast feeding for one month or more prevented food allergy at 3 years and respiratory allergy at 17 years of age, whereas at least six months of breast feeding was required to prevent eczema. ${ }^{32}$ In our study a reduction in wheezing, but not asthma, was seen in both the Nutramigen and the fully breast fed groups. The effect in the fully breast fed group might to some extent be explained by the capacity of breast milk to protect against infection. ${ }^{33}$ It seems, however, that the reduced antigen content in an extensively hydrolysed cows' milk based formula may contribute to a reduction in wheezing episodes in the first years of life. Until now no randomised study of the preventive effect of cows' milk hydrolysates has shown an effect on respiratory allergy. Whether there is such a protective effect similar to that of breast feeding ${ }^{31}$ remains to be seen in the long term follow up studies of the children in this randomised trial.

In summary, this study lends support to the allergy preventive effect of an extensively hydrolysed cows' milk formula (Nutramigen) as part of an allergy preventive programme for high risk infants.

The devoted work of the research nurses Lena Lindell, Karin Thorell, Gunnel Bergsten, and Birgitta Hjalmarsson and the dietitians Ingrid Torehov, Gunnel Jonsson, and Marie Lantz is gratefully acknowledged. The formulas were supplied by Mead ohnson, USA. The study was financially supported by BristolMyers Inc, the Swedish Medical Research Council (Grant No 7510), the National Association for the Prevention of Asthma and Allergy (RmA), the Queen Silvia's Jubilee Fund, and the Division of Research, Jönköping City Council.

1 Burney PGJ, Working Group in Sardinia 1990. Evidence for n increase in atopic disease and possible causes. Clin Exp an increase in atopic dise

2 Croner S, Kjellman, N-IM. Development of atopic disease in relation to family history and cord blood $\operatorname{IgE}$ evels-eleven years follow-up in 1654 children. Pediatr Allergy Immunol 1990;1:14-20.

3 Arshad SH, Stevens M, Hide BW. The effect of genetic and enviromental factors on the prevalence of atopic disorders at the age of two years. Clin Exp Allergy 1993;23:504-11.

4 Andrae S, Axelson O, Björkstén B, Fredriksson M, Kjellman N-I M. Symptoms of bronchial hyperresponsiveness in relation to enviromental factors. Arch Dis Child 1988;63: 473-8.

5 Björkstén B. Risk factors in early childhood for the development of atopic diseases. Allergy 1994;49:400-7.

6 Kjellman N-I M. Prediction and prevention of atopic allergy. Allergy 1982;37:463-73.

7 Zeiger RS. Dietary manipulations in infants and their mothers and the natural course of atopic disease. Pediatr Allergy ers and the natural course of ato

8 Kjellman N-I M. Atopic disease in seven-year-old children: incidence in relation to family history. Acta Paediatr Scand 1977;66:465-71.

9 Hattevig G, Kjellman B, Sigurs N, Björkstén B, Kjellman N-I M. Effect of maternal avoidance of eggs, cows' milk and fish during lactation upon allergic manifestations in infants. Clin Exp Allergy 1989;19:27-32.

10 Sigurs N, Hattevig G, Kjellman B. Maternal avoidance of eggs, cow's milk and fish during lactation: effect on allergic manifestations, skin prick tests, and specific IgE antibodies in children at age 4 years. Pediatrics 1992;89:735-9.

11 Zeiger RS, Heller S, Mellon MH, Halsey JF, Hamburger $\mathrm{RN}$, Sampson HA. Genetic and enviromental factors RN, Sampson HA. Genetic and enviromental factors affecting the development of atopy through age 4 in children of atopic parents: a prospective randomized study of food allergen avoidance. Pediatr Allergy Immunol 1992;3:

12 Zeiger RS, Heller S. The development and prediction of atopy in high-risk children: follow-up at age seven years in 
a prospective randomized study of combined maternal and infant food allergen avoidance. $\mathcal{F}$ Allergy Clin Immunol

1995;95:1179-90.
Fälth-Magnusson K, Kjellman N-I M. Development of atopic disease in babies whose mothers were receiving exclusion diet during pregnancy-a randomized study. $\mathscr{J}$ Allergy Clin Immunol 1987;80:868-75.

14 Fälth-Magnusson K, Kjellman N-I M. Allergy prevention by maternal elimination diet during pregnancy: a five yea follow-up study. F Allergy Clin Immunol 1992;89:709-13.

15 Arshad SH, Matthews S, Gant C, Hide DH. Effect of allergen avoidance on development of allergic disorders in infancy. Lancet 1992;339:1493-7.

16 Donally $\mathrm{HH}$. The question of the elimination of foreign protein (egg white) in woman's milk. F Immunol 1930;19: 15-40.

17 Kilshaw PJ, Cant AJ. The passage of maternal dietary proteins into human breast milk. Int Arch Allergy Appl

18 Jacobsson I, Lindberg T, Benediktsson B, Hansson BG Dietary bovine betalactoglobulin is transferred to human Dietary bovine betalactoglobulin is transferred to human
milk. Acta Paediatr Scand 1985;74:342-5.

milk. Acta Paediatr Scand 1985;74:342-5.

9 Cant AJ, Marsden RA, Kilshaw PJ. Egg and cow's milk hypersensitivity in exclusively breast fed infants with eczema, and detection of egg protein in breast milk. $B M Y$ 1985;291:932-5.

20 Vandenplas Y, Hauser B, Van den Borre C, Sacre L, Dab J. Effect of a whey hydrolysate prophylaxis of atopic disease. Ann Allergy 1992;68:419-24.

21 Businco L, Dreborg S, Einarsson R, et al. Hydrolysed cow's milk formulae. Allergenicity and use in treatment and prevention. An ESPACI position paper. Pediatr Allergy Immunol 1993;4:101-11.

22 Kjellman N-I M, Gustavsson M. Topical sodium cromoglycate in atopic dermatitis. A disappointing but informative trial. Allergy 1986;41:423-8.
23 Seymour JL, Keswick BH, Hanifin JM, Jordan WP, Milligan MC. Clinical effects of diaper types on the skin of the normal infants and infants with atopic dermatitis. $\mathcal{F} \mathrm{Am} \mathrm{Acad}$ Dermatol 1987;17:988-97.

24 Hattevig G, Kjellman B, Johansson SGO, Björkstén B. Clinical symptoms and IgE responses to common food proteins in atopic and healthy children. Clin Allergy 1984;14:551-9.

25 Dreborg S. Skin tests used in type I allergy testing. Allergy 1989;44(suppl 10):22-30.

26 Kjellman N-I M, Johansson SGO. Soy versus cow's milk in infants with a biparental history of atopic disease: development of atopic disease and immunoglobulins from birth to 4 years of age. Clin Allergy 1979;9:347-58.

27 Vandenplas Y, Hauser B, Van den Borre, et al. The long term effect of a whey hydrolysate formula on the prophylaxis of atopic disease. Eur F Pediatr 1995;154:488-94.

28 Høst A, Husby S, Østerballe O. A prospective study of cow's milk allergy in exclusively breast-fed infants. Acta Paediatr Scand 1988;77:663-70.

29 Oldæus G, Björkstén B, Einarsson R, Kjellman N-IM. Antigenicity and allergenicity of cow milk hydrolysates intended for infant feeding. Pediatr Allergy Immunol 1991;4:156-64.

30 Businco L, Cantani A, Longhi MA. Anaphylactic reactions to a cow's milk whey protein hydrolysate (Alfaré, Nestlé) in to a cow's milk whey protein hydrolysate (Alfare, Nestle) in
infants with cow's milk allergy. Ann Allergy 1989;62:333-5.

31 Rylander E, Pershagen G, Eriksson M, Nordvall L. Parental smoking and other risk factors for wheezing bronchitis in children. Eur f Epidemiol 1993;9:517-26.

32 Saarinen UM, Kajosaari $M$. Breastfeeding as prophylaxis against atopic disease: prospective follow-up study until 17 years old. Lancet 1995;346:1065-9.

33 Cunningham AS, Jelliffe DB, Jelliffe EFP. Breast-feeding and health in the 1980s: a global epidemiologic review. $\mathcal{F}$ Pediatr 1991;118:659-66. 